Questions vives

\section{Questions Vives}

Recherches en éducation

$n^{\circ} 21 \mid 2014$

Le travail collectif des enseignants en question(s)

\title{
Des dimensions collectives comme dimensions génériques du travail humain et leurs déclinaisons dans des activités d'enseignement partenariales
}

collective and generic dimensions of human work and their variations in partnership teaching activities

Patricia Champy-Remoussenard

\section{(2) OpenEdition}

Journals

Édition électronique

URL : http://journals.openedition.org/questionsvives/1534

DOI : 10.4000/questionsvives. 1534

ISSN : $1775-433 \mathrm{X}$

Éditeur

Université Aix-Marseille (AMU)

Édition imprimée

Date de publication : 15 septembre 2014

ISBN : 978-2-912643-45-2

ISSN : 1635-4079

Référence électronique

Patricia Champy-Remoussenard, «Des dimensions collectives comme dimensions génériques du travail humain et leurs déclinaisons dans des activités d'enseignement partenariales », Questions Vives [En ligne], nº 21 | 2014, mis en ligne le 15 septembre 2014, consulté le 01 mai 2019. URL : http:// journals.openedition.org/questionsvives/1534; DOI : 10.4000/questionsvives.1534

Ce document a été généré automatiquement le 1 mai 2019.

\section{(†)}

Questions Vives est mis à disposition selon les termes de la licence Creative Commons Attribution Pas d'Utilisation Commerciale - Pas de Modification 4.0 International. 


\section{Des dimensions collectives comme dimensions génériques du travail humain et leurs déclinaisons dans des activités d'enseignement partenariales}

Collective and generic dimensions of human work and their variations in partnership teaching activities

Patricia Champy-Remoussenard

\section{Introduction}

1 Les recherches en analyse du travail montrent que les dimensions collectives constituent une caractéristique quasi universelle du travail humain. On peut donc les considérer comme génériques et transversales. Elles trouvent des déclinaisons spécifiques dans des secteurs d'activité et des "métiers" différents. A l'heure où le travail humain se transforme de façon considérable et accélérée (Champy-Remoussenard, 2014), les dimensions collectives évoluent elles aussi et participent d'une transformation de métiers de la relation humaine, notamment des activités de ceux qui éduquent, forment, enseignent, accompagnent. En prenant appui sur les résultats des études ${ }^{1}$ sur les dimensions collectives dans l'ensemble des activités de travail, on renouvelle la compréhension des activités des enseignants, notamment dans leurs dimensions collectives en ce qu'elles recèlent de travail caché et difficilement formalisable. Nous montrerons tout d'abord que les dimensions collectives sont des dimensions génériques $\mathrm{du}$ travail humain, qu'elles participent de son caractère mal visible. Puis nous insisterons sur le statut qu'elles donnent à la relation à autrui, au lien qu'elles entretiennent avec le travail réel, aux processus de construction, de transmission, d'évaluation des compétences. C'est enfin sur cette base que la spécificité des dimensions collectives du 
travail des enseignants sera explorée, compte tenu de leur rapport spécifique à la reconnaissance des autres adultes, plus particulièrement dans les dispositifs partenariaux qui reconfigurent les formes traditionnelles du travail des enseignants.

\section{Les dimensions collectives dans le travail humain}

\subsection{Des dimensions transversales ou génériques}

2 La dimension est, au sens sociologique du terme, une composante d'un fait social. Dans le cas du travail, elle permet à la fois d'en prendre la mesure et de le situer dans un espace, c'est à dire de caractériser son étendue, son ampleur, ses limites, ses frontières. Elle renvoie aussi à la multiplicité interne des registres constitutifs de l'expérience de travail, qui peuvent donc être appréhendés selon différents niveaux d'analyse. En ces deux sens, le concept de dimension semble opérant pour tenter à la fois de resituer et de restituer, de comprendre, d'analyser le travail réel et ce qu'il s'y joue de collectif. À la suite de Schwartz $(1997,33)$ quand il écrit « La dimension collective apparaît comme un caractère constituant à peu près universel $l^{2}$ du travail ", nous l'envisagerons tout d'abord comme une des dimensions transversales ou génériques du travail humain. Nous ferons, aussi ici, l'hypothèse que l'exploration des dimensions collective est une clef d'analyse importante dans une perspective d'analyse de la complexité du travail.

Les dimensions collectives vues comme transversales constituent un des dénominateurs communs à l'ensemble des activités de travail, un aspect universel du vécu du travail humain (qui se trouvent déclinés dans la multitude des situations singulières), de ce qui est partagé et renvoie au fond universel de l'expérience de tous ceux qui travaillent. Ce qui est partagé n'est pas à entendre ici comme ce qui gommerait les individualités, les différences, les styles etc., mais bien comme ce qui permet à ces formes de singularité d'être exprimées, communiquées, partagées, échangées.

4 Les dimensions collectives génériques ou transversales définies ici sur un plan anthropologique seront donc distinguées du genre ou pôle générique de l'activité (Clot, 1999), du travail collectif ou " partagé » (Marcel, 2009), des collectifs ou groupes de travail (Trognon, Dessagne, Hoch, Dammerey \& Meyer, 2004), formes collectives qui s'inscrivent dans des groupes professionnels ou dans le travail déployé à l'intersection des activités de ces groupes.

5 La distinction entre « genre » et «style », proposée par Yves Clot (1999), participe d'une prise en compte du collectif dans la perspective d'une analyse psychologique de l'activité de travail. Le genre est une "composante impersonnelle» et "générique " de la compétence, de l'ordre d'une "culture professionnelle collective" où se "marient subtilement règles formelles et informelles ». Selon Clot, « un genre relie toujours entre eux ceux qui participent à une situation, comme des co-acteurs qui connaissent, comprennent et évaluent cette situation de la même façon ». C'est encore, le « champ des sous-entendus sociaux indispensables pour travailler ». Le genre définit donc le rapport au collectif et oriente l'activité singulière, c'est un "instrument d'action», et une " condition de l'anticipation de l'activité de l'autre et donc de l'échange social» (Clot, 1999, p. 98). Il peut être alors compris comme ce qui peut relier des vécus professionnels inscrits dans des domaines d'activité déjà repérables, repérés, et délimités pour leurs dénominateurs communs. 
6 (Trognon et al., 2004) introduisent, eux, le terme de «collectif de travail» et le distinguent de celui de groupe de travail. Un groupe de travail est formel et «la participation [...] n'est pas volontaire mais imposée par l'organisation " tandis qu'un collectif est informel, c'est-à-dire "créé par les membres eux-mêmes, pour combler l'écart entre ce qui est attendu de l'équipe et ce qui est réellement faisable dans la situation » (p. 418). Enfin, pour Marcel et Garcia (2010), le «travail partagé » suppose un partage des activités avec un objectif commun. Il s'agit de faire ensemble pour réussir ensemble.

7 La compréhension des questions relevant du collectif au travail peut donc être étayée à plusieurs niveaux et à l'aide de plusieurs concepts. Le concept de dimension générique utilisé dans une perspective anthropologique et sociologique (au niveau générique), le concept de genre associé à celui de style (Clot, 1999), ceux de travail partagé (Marcel, Garcia 2010), de groupe et de collectif de travail dans une perspective clinique et d'analyse des pratiques. Mais, quel que soit le point de vue adopté, la relation à autrui dans l'activité humaine définit les dimensions collectives.

\subsection{Le travail humain et la relation à autrui}

Si on répond par la négative à la question : « il y a-t-il un travail humain qui ne soit pas collectif?», on postule que l'humanité du travail réside dans le fait que l'activité de travail relie d'une manière ou d'une autre un individu aux autres. Même quand elle s'effectue dans l'isolement - d'un point de vue spatial - dans la mesure où elle est définie par sa valeur et son utilité sociale, elle se trouve reliée à autrui. Canguilhem spécifie le travail humain, en le distinguant du travail des machines en termes de "relations à ». Selon lui, « les machines dites intelligentes sont des machines à produire des relations entre les données qu'on leur fournit mais elles ne sont pas en relation à ce que l'utilisateur propose à partir des relations qu'elles engendrent pour lui » $(2005$, p. 27). Oddone (1981, p. 25) remarque, en mettant en œuvre la méthode des « instructions au sosie ", que « le problème du rapport à la tâche ne peut se résoudre qu'en modifiant les rapports aux camarades de travail, aux organisations syndicales (et de partis) et à la hiérarchie ». La relation à autrui constitutive du travail est également particulièrement mise en exergue par Clot, qui lui donne une grande place dans la clinique de l'activité. Selon lui, «l'action du sujet doit traverser le milieu mouvant des activités d'autrui », et dans un processus d'adressage, "l'activité du sujet n'est pas uniquement tournée vers l'objet de la tâche mais tout autant vers l'activité des autres portant sur cette tâche et vers ses autres activités à lui »(Clot, 1999, p. 98).

9 Selon Clot et Leplat, l'activité est d'ailleurs à la fois personnelle (de façon irréductible), interpersonnelle puisque "dans chaque situation singulière car sans destinataire, l'activité perd de son sens", transpersonnelle car "traversée par une histoire collective ", impersonnelle " sous l'angle de la tâche prescrite (Clot \& Leplat, 2005, p. 310). Durrive dans son essai de reconceptualisation de la compétence (2013, p. 191) propose d'« envisager le travail en fonction des autres». Avec l'outil ergologique "tâche/ service ", il propose un modèle de l'agir en projet d'usage de soi, avec un triple positionnement : par rapport aux autres (le service à rendre), par rapport au monde réel qui me résiste (la tâche à accomplir) et par rapport à soi-même (les ressources que je dois mobiliser) (Durrive, 2013, p.180). Incontournable, omniprésent, le travail collectif 
échappe pourtant bien souvent à l'analyse, et c'est sans doute par ce que, ce qui reste profondément obscur, c'est la relation entre l'acte et l'acteur (Durrive 2013, p. 170).

Émanant de cadres conceptuels différents ces travaux relatifs à l'activité humaine au travail mettent tous l'accent sur cette relation irréductible entre soi au travail et autrui. Cette relation s'inscrit elle-même dans ce qui fait le travail réel, dans la tension entre tâche et activité définie par l'ergonomie (Leplat, 1997).

\subsection{Dimensions collectives et travail réel}

11 Les dimensions collectives du travail sont associés à la nature même du travail réel : « Ce qui est analysé à la loupe du travail réel va de pair avec la détermination de ce qui est collectif dans le travail » (Schwartz, 1987, p. 188). Car c'est « le groupe qui dirige et gère qui doit parer aux innombrables difficultés qui naissent du décalage existant de fait entre les prévisions et la réalité » (Oddone, 1981, p. 25).

L'organisation du travail entend régir une partie des interactions dans les collectifs. Les prescriptions indiquent donc au moins en partie ce que devrait être le travail d'un groupe. Cependant et paradoxalement, les recherches montrent que les «rapports de coopération » ne peuvent, en fait, pas être prescrits (Schwartz, 1997). Cette impossibilité signifie donc que le collectif est doté d'autonomie et que l'organisation collective réelle du travail est en tension avec l'organisation du travail prescrite. D'après Six (2002), le travail en collectif permettrait d'ailleurs, tout particulièrement, de s'émanciper de la prescription.

La méthode de l'écriture sur l'activité (Champy-Remoussenard, 2009 ChampyRemoussenard \& Starck, 2011) qui fonde l'analyse de situations de travail sur l'exploration de récits écrits relatant le travail réel et mis en discussion dans des groupes a permis de mettre en évidence l'importance des dimensions collectives comme voies d'accès et, pourrait-on dire, descripteurs et analyseurs du travail réel ${ }^{3}$. Dans les groupes d'écriture sur l'activité, l'écriture devient une écriture impliquée, habitée par l'acteur et donnant accès aux situations de travail réelles, particulièrement lorsqu'elle situe l'acteur dans le collectif dans lequel ont eu lieu ses activités. À cet égard, le texte d'une institutrice, écrit sous forme de liste à partir de la répétition du déclencheur «si seulement j'avais pensé a ${ }^{4}$ ", met, par exemple, bien en évidence à quel point le travail quotidien est défini par l'intrication entre la succession des activités, les préoccupations personnelles de l'enseignante et les interactions avec un ensemble d'acteurs qui constitue son collectif de travail (les parents de ses élèves, son propre environnement familial, un représentant de commerce, l'une de ses collègues, le responsable d'une compagnie de bus...). Les exemples de ce type sont innombrables. La plupart des textes rédigés par les professionnels font une place essentielle à la relation des acteurs à ce collectif. A l'occasion d'une écriture vive et impliquée, les dimensions collectives du travail sont comme naturellement exprimées.

Dejours (2009) et d'autres chercheurs en psychodynamique du travail insistent, eux aussi, sur l'écart entre l'organisation prescrite et l'organisation réelle du travail, en analysant dans ce cadre les stratégies complexes mises en œuvre par le collectif pour rendre le travail supportable aux individus. Durat qui a étudié le rôle du collectif de travail dans le cas des risques psychosociaux dans l'enseignement supérieur (2013) cite Kirouac (2011). Celui-ci montre que dans les cas d'épuisement professionnel, les conflits interindividuels, les facteurs psychosociaux ne sont pas du seul ressort de l'individu alors que les 
interventions pratiquées auprès de personnes en situation d'épuisement s'en tiennent la plupart du temps au seul périmètre de la psychologie et de la responsabilité individuelle. D'autres travaux (Loriol, 2000) montrent comment les collectifs qui expriment le moins de stress sont ceux qui réalisent un travail collectif de partage à propos des difficultés rencontrées sur le terrain.

Les dimensions collectives permettent donc de comprendre les modes d'organisation effectives du travail et les ressorts d'un travail qui procure satisfaction et santé. Les collectifs soudés apparaissent comme véritables supports de la qualité et de la satisfaction, voire de la sérénité au travail (Durat, 2013). Mais, le travail collectif s'il tire de la force de la cohésion qu'il suscite génère aussi des conflits. Travailler ensemble, ce n'est pas forcément être bien ensemble. Les controverses, les disputes naissent du collectif et peuvent tout à la fois empêcher de continuer à travailler ensemble et contribuer à la construction et à la stabilité du collectif. La possibilité d'exprimer les débats internes aux milieux professionnels participe fondamentalement de la vie du collectif. L'échange entre ses membres est un élément important de la vie du collectif. Il favorise le collectif et peut être formel ou informel. Il requiert la confiance (Dejours, 2009, p. 78). Si le temps et l'espace dialogique ne peuvent être rendus obligatoires ils peuvent être recommandés. En effet, s'il est déficitaire ce registre de l'échange à propos de l'activité est pathogène (Dejours, 2009). L'échange entre professionnels, organisé selon certaines modalités débouche sur une meilleure connaissance du collectif et, s'il est poussé assez loin sur un savoir analyser collectif.

\subsection{Collectif, compétences, évaluation et transmission de l'expérience}

16 Le processus de transmission de l'expérience qui participe de la fonction éducative du travail implique de manière incontournable la dynamique collective. Même médiatisée (dans le cas des didacticiels et des outils de simulation), l'expérience nécessite d'être exprimée par les uns pour être transmises aux autres. La formation des novices ou des nouveaux est donc un des aspects de la vie collective au travail, qu'elle se décline selon des modalités formelles ou informelles. Des travaux sur les activités en cours de professionnalisation des « emplois-jeunes » ont montré que, même dans un contexte très spécifique (absence de collectif constitué, absence de modèle de la compétence, pour le débutant, position d'empiètement inévitable sur les territoires des autres professionnels, etc.), le rapport au collectif (dans ce cas atypique et bricolé à l'initiative du jeune) reste un élément fondamental du processus du construction des compétences (ChampyRemoussenard \& Meyer, 2004).

Le processus de construction et de transmission de l'expérience pose de manière spécifique les questions liées aux dimensions collectives du travail, au travail collectif et aux collectifs de travail. L'expérience nécessite d'éprouver ce qui n'est pas encore connu et l'ajuster à ce qui est déjà connu. C'est en puisant dans l'expérience déjà élaborée que se renouvelle le capital d'expérience du collectif en fonction des transformations du contexte et donc des situations, sur la base d'un échange au sein du collectif qui suppose des formes de relations entre anciens et nouveaux. Dans ses comptes rendus d'instructions au sosie (Oddone, 1981), l'ouvrier Luigi dit: «En général, c'est de l'expérience de ceux qui étaient là avant vous dont on se sert. Et, une fois qu'on a compris 
qu'il existe des tours de main, on fait tout ce qu'il faut pour découvrir ceux qui conviennent à la situation. »

Si l'expérience est issue d'un processus collectif et organisationnel, il est intéressant de ré examiner d'un œil critique l'acception individuelle de la manière dont elle s'actualise dans la compétence. "Elle met en jeu un collectif d'acteurs situé dans un cadre organisationnel " et, selon lui, "même des activités qui paraissent essentiellement individuelles relèvent peu ou prou de normes d'organisation» (Lorino, 2014, p. 209). La « capacité de faire dépend étroitement, non seulement de celui qui fait mais de tous ceux auxquels celui qui fait s'adresse par son action » (Lorino, 2014, p. 211).

C'est d'ailleurs lorsqu'on cherche à analyser le travail en termes de compétences que les dimensions collectives s'imposent à la fois comme une évidence et comme une difficulté. Durrive souligne les problèmes posés par les modèles actuels de gestion des compétences: "on a d'un côté l'usage, parfois intempestif, de grilles anonymes pour cerner la compétence à travers une codification (une désincarnation qui incite certains à parler de déshumanisation, comme nous l'avons vu) et d'un autre côté, un appel continu à l'engagement personnel, l'injonction à l'initiative et à l'innovation dans le travail » (2013, p. 160).

L'idée de compétence conduit à montrer ce qui fait la différence entre les individus (Vergnaud, 1995). La plupart des démarches d'évaluation des compétences pratiquées dans les organisations le sont dans le but de repérer des compétences individuelles et de proposer une reconnaissance individualisée des résultats du travail fourni par les acteurs (par un système de primes ou d'avancement individuel par exemple). C'est encore le cas dans les Bilans de Compétences où, à nouveau, il s'agit de mettre en évidence les compétences dont disposent des individus. Même organisés sous forme collective, ils s'inscrivent toujours dans une fonction d'ajustement individuel entre les compétences disponibles et les compétences recherchées. Ainsi, dans les pratiques de VAE, c'est l'expérience construite au cours d'un parcours singulier qui doit être formalisée et rendue comparable aux compétences visées dans référentiel.

21 Dans tous ces cas, la dimension collective n'est nullement niée. Savoir travailler avec d'autres est une des facettes des compétences qui est appréhendée, mais la façon dont le rapport au collectif définit chacune des situations du travail réel reste dans l'ombre, parce que l'évaluation, le bilan, la validation sont de nature individuelle et visent des objectifs et des usages individuels.

22 Si les dimensions collectives sont communes à tout type de travail humain, le collectif revêt des caractéristiques particulières dans les métiers du service et de la relation à autrui (Piot, 2009). Cet ensemble d'activités centrées autour de leur rapport à un usager ou à un client qui s'est fortement développé pendant ces dernières décennies offre de nouvelles perspectives pour l'étude des dimensions collectives du travail. Elles montrent comment le collectif agit, y compris dans les contextes où le travail semble se dérouler dans un fort isolement. Les évolutions des activités enseignantes sont un des secteurs d'activité dans lequel se dessinent actuellement des évolutions du rapport au collectif dans les activités de travail. 


\section{Les dimensions collectives du travail dans les activités d'enseignement}

\subsection{Une lente prise en compte de la question du collectif dans les travaux de recherche}

23 La prise en compte des dimensions collectives du travail a longtemps été plutôt négligée dans la plupart des recherches portant sur les enseignants d'ailleurs souvent caractérisés par le côté individualiste de leur travail. Rochex et Kherroubi (2004) ont montré qu'alors que des travaux indiquaient que la mobilisation collective semble «faire émerger un rapport au métier plus positif» peu de recherches «empiriques portaient sur cette dimension collective». Un net développement des recherches en éducation sur ces questions caractérise la période la plus récente.

Un certain nombre des entrées analytiques que nous venons de présenter peuvent être pertinentes pour faire progresser la connaissance des dimensions collectives dans le travail enseignant. En parlant des «métiers» de l'enseignement, nous prenons la précaution de ne pas amalgamer tous les enseignants, qui exercent de fait dans des contextes et avec des publics variés. Les activités d'enseignement varient en fonction de nombreux paramètres. Nombre des remarques que nous allons faire sont donc à relativiser en fonction des contextes d'activité et seraient à mettre à l'épreuve de données empiriques. Nous distinguerons bien ici l'enseignant du formateur (Durrive, 2013) avec l'hypothèse que deux caractéristiques de l'activité de la majeure partie des enseignants spécifient particulièrement leur rapport au collectif : leurs relations respectives avec les adultes et avec les enfants; le travail dans la classe et hors la classe.

\subsection{Collectifs souhaités et collectifs contraints}

Si le travail collectif est incontournable et s'il ne peut être véritablement prescrit, il fait pourtant bel et bien l'objet, dans le cas des enseignants, d'incitations, de demandes institutionnelles, notamment à l'occasion de la mise en place de nouveaux dispositifs (Marcel Dupriez \& Périssed-Bagnoud, 2007). Un des axes de recherche sur ces questions porte sur la nature de l'engagement et sur la contrainte et l'initiative en matière de fonctionnement collectif. S'il y a nécessairement une tension entre le fonctionnement collectif prévu et le fonctionnement collectif effectif, c'est aussi l'impact d'un engagement contraint et d'un engagement à l'initiative des acteurs qui a été étudié. Les travaux mettent d'ailleurs l'accent sur la distinction entre collectif organisé sur un mode formel et sur un mode informel. Cette analyse débouche sur la proposition de distinguer la notion de communauté de pratiques et celle de communauté de processus. Alors que dans une communauté de pratiques, les membres sont unis par le type de solidarité qui unit les membres d'une tribu (solidarité mécaniste au sens de Durkheim), les interactions entre acteurs dans une communauté de processus sont contraintes et relèvent d'une solidarité « organique » au sens de Durkheim (Lorino, 2014).

Ces deux questions - du formel ou de l'informel, de la contrainte ou du choix - parmi d'autres que nous ne pouvons présenter ici montrent que ce sont les situations qui peuvent permettre de comprendre les dimensions collectives. L'analyse de celles dans lesquelles se trouvent impliqués les enseignants - situations dans la classe et situation 
hors de la classe - permet actuellement d'avancer quelques hypothèses sur ce qui détermine le rapport au collectif dans les métiers de l'enseignement. C'est sur ce point que nous voudrions insister dans cette deuxième partie.

\subsection{Des professionnels en quête du regard des autres adultes}

27 Les dimensions collectives dans le travail supposent le regard de l'autre. Travailler c'est agir sous, avec et peut-être par le regard de l'autre (celui qui partage le même métier mais l'exerce à sa façon, celui qui exerce son travail dans d'autres métiers). Or, c'est principalement sous le regard des enfants ou des adolescents que travaillent les enseignants. Ce qui leur manque ou qui n'est (et surtout n'était pas là), sauf dans certaines situations spécifiques, c'est le regard des autres enseignants et plus généralement des autres adultes: "Dans son travail, l'enseignant se trouve être le représentant isolé du groupe d'adultes face à la classe, groupe d'égaux auquel il n'appartient pas » (Abraham, 1982, p. 53).

28 Faire voir son travail participe d'une quête de reconnaissance et de légitimité. Seul adulte dans sa classe et donc dans la plupart de ses situations de travail, l'enseignant souffre d'un déficit de visibilité, de reconnaissance et même de connaissance de son métier. Or, le degré de visibilité participe de la construction de l'image professionnelle de soi qui est déterminée par la considération qu'il a de lui-même et par celle qu'il attribue aux autres à son égard (Abraham, 1982, p. 38).

29 Mais le regard de l'autre, s'il rompt la solitude et constitue un appui, une réassurance et une source de reconnaissance est aussi une menace potentielle, une intrusion dans l'intimité du travail. Le regard de l'autre, la visibilité de l'activité sont donc à la fois recherchés et craints. C'est dans cette tension que s'inscrit le rapport au collectif et la quête du regard de l'autre. Ce rapport en tension dont on peut faire l'hypothèse qu'il caractérise là encore le travail en général concerne tout particulièrement l'activité des enseignants dans l'organisation scolaire "classique». Alors même que le regard des autres est nécessaire, il peut gêner et se trouver rejeté si sa portée est exclusivement évaluative et s'il constitue une menace. Par exemple, si l'activité du formateur d'enseignants est trop tournée vers sa fonction évaluative (Zeitler, 2012) et les formes de mise en concurrence, elle inhibe les « apprentissages interprétatifs » de l'enseignant en formation. Et plus généralement, l'excès d'évaluation peut constituer un frein à un fonctionnement collectif marqué par le besoin de confiance, de solidarités collectives et de loyauté.

\subsection{Les effets du travail enseignant hors de la classe (Marcel \& Piot, 2005)}

Les pratiques des enseignants hors de la classe - celles qui les font sortir de la classe nous semblent de nature à modifier ce déficit de reconnaissance. Elles sont également susceptibles de modifier le regard des enseignants sur leur propre travail et leurs pratiques elles-mêmes. Les contextes de travail partenarial, en équipe de projet ${ }^{5}$, etc. sont donc particulièrement intéressants à étudier. Dans ces situations, enseigner, ce n'est plus seulement faire classe, faire l'enseignant dans la classe mais le faire ailleurs, autrement, avec d'autres adultes sous le regard d'autres adultes. 
31 Le travail collectif hors la classe implique des situations qui débordent et remettent en cause le cadre scolaire, l'échange en huis clos entre un enseignant et un groupe d'élèves, la porte fermée que l'on n'ouvre et ferme qu'en début et fin de cours. Une recherche conduite sur les stages d'un an en entreprise proposés à des enseignants d'économie/ gestion (Champy-Remoussenard \& Deville, 2013) montre qu'un des effets perçu comme gratifiant de leur séjour dans l'entreprise correspond à la possibilité par les enseignants de voir leurs compétences reconnues par les professionnels des entreprises dans lesquelles ils travaillent. Dans leur quotidien habituel, la plupart des enseignants exposent leur activité au regard de leurs seuls élèves.

Le collectif des élèves est une des dimensions centrales du travail des enseignants. Le face à face pédagogique a été largement étudié sous l'angle des interactions entre un individu adulte enseignant et un groupe d'élèves (enfants ou adolescents). Les travaux sur le fonctionnement des enseignants dans des collectifs d'adultes montrent que l'implication dans un collectif d'adultes a également un impact sur le rapport au collectif des élèves (Marcel \& Garcia, 2010).

\subsection{Le travail avec d'autres professionnels dans le cadre d'une mission éducative partagée}

On peut définir à l'instar de Marcel et Garcia (2010, p. 28) le travail partagé comme «l'ensemble des activités professionnelles de l'enseignant mettant en scène plusieurs adultes (autres enseignants, partenaires), selon des modalités individuelles ou collectives », avec un objectif commun « faire ensemble pour réussir ensemble ». L'auteur remarque que «le travail partagé n'est pas une modalité de travail enseignant supplémentaire qui viendrait s'ajouter au travail traditionnel de l'enseignant seul dans sa classe » (Marcel \& Garcia, 2010, p. 29). Il s'agit donc bien de formes du travail enseignant qui se développent en lien avec des partenaires et des objectifs spécifiques, cohabitant en quelque sorte avec la forme traditionnelle du travail en salle de classe avec les seuls élèves. Ce travail partagé semble tout particulièrement s'émanciper de toute forme de prescription et se déployer de manière privilégiée - ce qui peut apparaître comme un paradoxe ou comme quelque chose de tout à fait logique - en marge de l'institution scolaire. Cette analyse, si elle s'applique à toute forme de travail partagé, implique d'ailleurs que même dans le travail partagé subi, l'enseignant ne puisse se borner à appliquer la prescription.

Une étude conduite à propos d'un dispositif visant l'éducation à l'esprit d'entreprendre et dénommé « Mini-Entreprises» (ME) servira ici d'analyseur de ce qui se joue dans une de ces situations de "travail partagé ». Plusieurs chercheurs des trois équipes internes du Centre Interuniversitaire de recherche en éducation de Lille a en effet été sollicitée pour enquêter sur ce dispositif ${ }^{6}$ mis en œuvre dans l'académie de Lille depuis plusieurs années. Les travaux réalisés s'inscrivent dans un programme de recherche plus large qui étudie les relations écoles-entreprises dans de multiples contextes et différents dispositifs ${ }^{7}$. Il s'agit d'analyser divers outils, pratiques, dispositifs de formation qui visent à rapprocher l'école et les entreprises, améliorer l'orientation et l'insertion professionnelle des jeunes, faire progresser la connaissance que les enseignants et autres acteurs du système éducatif ont $\mathrm{du}$ monde du travail, plus globalement inscrits dans une intention sociale de professionnalisation (Wittorski, 2007; Champy-Remoussenard, 2010). Les minientreprises sont des entreprises créées pour une durée d'un an par une équipe 
constituées d'élèves, d'enseignants, de marraines et parrains d'entreprises (tous volontaires et le plus souvent impliqués hors du temps scolaire obligée et des programmes) et de partenaires associatifs. Ce travail collectif est orchestré sur la base d'une pédagogie du projet) et se fait sur la base de l'engagement choisi des acteurs. Il vise l'atteinte de la compétence clef « esprit d'initiative et d'autonomie » du socle commun et s'inscrit dans ce qu'on peut appeler l'éducation à Champy-Remoussenard et al. (2012) ; Zaid, Champy-Remoussenard(2013). l'entreprenariat ou à l'esprit d'entreprendre ${ }^{8}$.

L'enquête sur le terrain a été menée entre septembre 2012 et mars 2014. Des entretiens semi-directifs ont été menés auprès de 10 enseignants, de 11 élèves, de 6 «pilotes » du dispositif. Des observations ont été réalisées dans quelques ME ou lors de manifestions ou actions ponctuelles (concours régional, journée de préparation au concours dite «J-50: les mini font leur buzz ", journée de formation des enseignants, séminaires de créativité et challenge " 30 heures pour créer " destinés à initier la ME). Des observations plus régulières auprès de $2 \mathrm{ME}$ concernant des élèves et des enseignants de collège ont été menées d'octobre 2012 à mai 2013. Un questionnaire élaboré au cours de l'enquête a été adressé à plus d'une centaine d'enseignants après le concours régional, soit à la quasitotalité des référents des ME pour la session 2012-2013. L'ensemble des dossiers déposés par les ME pour le concours régional ont été collectés et ont fait l'objet d'analyses spécifiques.

Les effets de ce travail impliquant un partenariat entre adultes et enfants dans une logique de fonctionnement en projet qui constituaient l'objectif visé par cette recherche sont multiples. Les propos d'un directeur d'association partenaire d'un Rectorat sur ce dossier sont éloquents. Le dispositif crée selon lui « du lien entre enseignants et élève ", en « cassant la relation très descendante entre l'enseignant et l'élève, l'enseignant qui sait et les élèves qui ne savent pas (...) qu'on a tous mal vécu à l'école ». D'autre part, quand on lui pose la question de la portée du dispositif en termes de relations écoles/entreprises, il signale un "rapprochement entre les profs... qui se comprennent mieux », et le fait que les chefs d'entreprise parrains/marraines des Mini-entreprises se "prennent une claque » et se rendent compte que le «métier d'enseignant, c'est pas simple». Le travail commun est présenté comme un moyen de " réduire les idées reçues » ${ }^{9}$. Les enseignants sont parfois présentés dans leurs formes d'engagement dans le dispositif (sur la base d'un volontariat) comme peu autonomes, voire dépendants des animateurs de l'association et surtout prisonniers de la forme scolaire, de la logique des programmes et des référentiels et d'une culture de la réussite obligatoire. Mais dans la plupart des cas, l'enquête montre au contraire que la ME est perçue comme la possibilité de se mettre à distance de son identité professionnelle habituelle et de tester une autre identité, une autre manière d'agir. Les enseignants en dirigeant les actions de la ME, en travaillant aux côtés des minientrepreneurs pour sortir le produit, en cherchant de nouvelles sensations dans leur cadre professionnel visent à élargir leur expérience qui ne peut plus être considérée ici comme strictement professionnelle.

Dans ce cas de figure, le partenariat est considéré comme une dynamique d'influence et de formation destinée à sortir les enseignants de "la dynamique scolaire ", "sortir du cadre scolaire " par le moyen de la dynamique de travail en projet. Et si cet effet est recherché par les acteurs de l'association partenaire, il semble l'être aussi par les enseignants qui, pour certains d'entre eux semblent engagés dans un processus quasi irréversible créant une sorte de dépendance au plaisir ressenti dans l'aventure : «le prof qui envoie un message un samedi matin, c'est assez rare ! Et là, il a envoyé un message en 
disant, c'est la plus belle rentrée scolaire que j'ai jamais vécue. Et là, je me suis dit « il y a un truc qui se passe ». Il dit aujourd'hui « sans mini-entreprise, je ne prends plus aucun plaisir à exercer mon métier ».

La division du travail entre enseignants et prestataires de l'association (qui sont pour certains salariés pour certains bénévoles) recèle une forte complexité et des tensions évidentes. Questionné sur le coût du dispositif, le directeur de l'association reconnait la part importante associée aux déplacements des accompagnateurs de l'association sur la région et appelle de ses vœux la plus grande autonomie des enseignants qui permettra de réduire les interventions de l'association. On voit aisément à quel point cet objectif met en tension l'association entre sa logique de développement liée aux besoins de l'Éducation Nationale et une logique de repli supposée dépendre de la plus forte implication des enseignants. La mission éducative partagée et le partenariat s'inscrivent, dans ce cas comme dans d'autres, également dans un marché de l'éducation. Dans le cas des minientreprises et de l'éducation à l'entreprenariat, la division du travail entre les différents partenaires relève d'une distribution de parts de marché et de la volonté des acteurs de l'éducation nationale de contrôler la qualité et les valeurs engagées par le travail des uns et des autres. Ces propos d'un chargé de mission École/entreprise le montrent : «Ce que j'espère c'est qu'on puisse réussir à faire travailler tous les opérateurs avec le même objectif sans concurrence, mais plus en complémentarité. Et je pense que si on arrive à faire ça, on arrivera à donner une cohésion à l'ensemble du processus. C'est une volonté du Rectorat. On sait qu' $\mathrm{X}^{10}$ seul ne pourra jamais tout faire, donc il faut vraiment que tous les opérateurs se situent, se positionnent et puissent agir en cohérence ». Le recours aux «opérateurs" qui participent ici autant à la formation et à l'accompagnement des élèves que des enseignants au cours du projet nécessitent, avec la forte montée en puissance du dispositif ME dans l'académie, des formes de régulation et de formalisation du travail collectif. Ainsi, un processus de labellisation des outils pédagogiques proposés par les opérateurs externes a été mis en place dans l'académie et trois « chargés de mission » assurent la promotion du dispositif et la mise en lien entre les opérateurs et les établissements.

Enfin le travail collectif effectué autour des ME produit un véritable collectif engagé autour d'un travail partagé. Évoquant le moment de la remise des prix qui vise à récompenser les meilleures mini entreprises, le même traduit sa perception du travail éducatif partagé par les différents acteurs: "On voyait qu'il se passe quelque chose de commun, que les gens sont réjouis de s'être engagés. En tout cas, c'est comme ça que je l'ai ressenti. Si j'avais une image à montrer, ce serait plus cela, cette communauté d'engagement, cette communauté de bonheur. On voit qu'il se passe quelque chose, de positif en plus. Avec des gens qui, il y a peu de temps, n'auraient pas pu se dire : « on est sur le même projet, contents d'être ensemble, entre l'entreprise, l'école ». Ce dispositif est bien ici considéré par les deux interviewés comme un moyen de faire évoluer les pratiques enseignantes. Le travail partagé y constitue bien globalement un levier de changement.

41 Ce type de dispositifs pourrait être étudié à l'aide de la théorie du "système d'activité " (Leontiev, 1959) qui permet, comme le propose Lorino de combiner des métiers distincts. Proche selon lui du « processus » en gestion, le système d'activité recouvre «l'ensemble des opérations, exécutées par une multiplicité d'acteurs appartenant à une pluralité de métiers, qui permet de fournir un résultat donné» (Lorino, 2014, p. 215). Ce cadre d'analyse incite à étudier l'activité enseignante en la resituant dans le système d'activité 
plus large dans lequel elle est incluse, en dépit des nombreux moments où l'enseignant est seul, pendant ses activités de préparation pédagogique ou exclusivement avec ses élèves, dans la classe. A l'heure où de nombreuses activités en partenariat se développent, cette voie nous parait donc particulièrement riche pour développer des analyses sur le travail des enseignants. Comme le montre Lorino (2014, p. 217), toute transformation de pratique dans un métier envisagé dans une communauté de pratique, interpelle les autres métiers, engagé dans le même processus et donnera du grain à moudre à la communauté de processus ». Une des voies d'analyse à venir semble donc bien consister à avancer vers une théorisation globale du travail enseignant qui inclut le travail partagé (Marcel \& Garcia 2010).

\section{Conclusion et perspectives}

42 Les pratiques qui se déploient dans une dynamique partenariale et qui engagent les enseignants aux côtés d'autres professionnels chargés de missions éducatives peuvent être saisies comme analyseur d'une reconfiguration collective du travail éducatif puissance de changement et d'innovation, de renormalisation accélérée, et de gain en pouvoir d'agir.

C'est dans ce sens qu'Abraham appelait de ses vœux en 1986 la création de réseaux de communication ouverte entre les différents groupes agissant dans le système éducatif comme un moyen pour améliorer les conditions « aliénatives » de l'enseignement.

Ces nouvelles formes de travail collectif interpellent en effet les représentations du travail des enseignants (est-il travail d'enseignement, travail éducatif, travail d'accompagnement?). Elles ont un impact sur le sentiment d'enfermement et d'isolement, le déficit de reconnaissance, les difficultés associées aux évolutions de carrière des enseignants, les velléités réaffirmées de "sanctuarisation » de l'école. Cet impact est cependant encore mal mesurable et, surtout, les changements notés ne concernent souvent qu'une partie marginale des enseignants d'un point de vue quantitatif. L'effet « boule de neige » ou autrement dit les retentissements sur le reste de la population enseignante restent à mieux cerner.

Pour cela, il nous semble qu'il faudrait, accorder une attention particulière aux situations de travail qui conduisent les enseignants à travailler avec d'autres adultes, en les étudiant à deux niveaux :

- Celui des dimensions génériques du travail qui sont présentes dans les pratiques enseignantes comme dans toutes les autres activités, ce qui leur permet tout particulièrement d'interagir avec d'autres professionnels dans le cadre d'un travail collaboratif ;

- Celui des dimensions relevant d'un genre professionnel, celui des enseignants, en relation plus ou moins harmonieuse avec des types d'activité et s'inscrivant dans différentes formes d'organisation collective du travail.

Starck, S., \& Deville, J. (2014). Se développer entre épreuve instituée et épreuve de la vie. Le cas des stages d'enseignants en entreprise, Chemins de formation, $n^{\circ} 18$. 


\section{BIBLIOGRAPHIE}

Abraham A. (1982). Le monde intérieur des enseignants, Paris : EAP.

Durrive, L. (2013). Compétences et activité de travail. «L'art de gérer ses débats de normes »? Synthèse de recherche en vue de l'HDR. Université de Strasbourg, LISEC, non publié.

Durat, L. (2013). De la formation des compétences des managers à l'apprentissage en situation critique. Rapport d'Habilitation à Diriger des Recherches, Université de Strasbourg, non publié.

Canguilhem, G. (2005/1943). Essai sur quelques problèmes concernant le normal et le pathologique (1943), réédité sous le titre Le Normal et le Pathologique, augmenté de Nouvelles Réflexions concernant le normal et le pathologique (1966), $9^{\mathrm{e}}$ rééd. Paris : PUF/Quadrige.

Champy-Remoussenard, P. (2009) Caractéristiques et fonctions de l'écriture sur l'activité professionnelle : l'éclairage des pratiques de VAE en France. In F. Cros, L. Lafortune, M. Morisse (Dir.), Les écritures en situations professionnelles (pp. 73-94). Presses Universitaires de l'Université du Québec, col. Éducations-Interventions.

Champy-Remoussenard, P. (2014). La construction de l'expérience dans une société en évolution accélérée, Recherche \& formation, 73, 91-102.

Champy-Remoussenard, P., \& Deville, J. (2013). La professionalidad de los docentes de secundaria en la prueba de la experiencia de « estancias de larga duracion » en una empresa. Barcelona, Universitat Internacional de Catalunya. UIC Publicacions, 209-218.

Champy-Remoussenard, P., Meyer, V. (Dir.) (2004). Etre emploi-jeune et après ? Nancy : PUN.

Champy-Remoussenard, P., \& Starck, S. (2011). Échanges écrits sur l'activité entre responsables de l'inspection de l'éducation nationale : quelles perspectives de Professionnalisation ? In M. Morisse, L. Lafortune, F. Cros (Dir.), Se professionnaliser par l'écriture, quels accompagnements? (pp. 181-199). Presses Universitaires du Québec.

Clot, Y. (1999). La fonction psychologique du travail. Paris : PUF, coll. Le Travail Humain.

Clot, Y., \& Leplat J. (2005). La méthode clinique en ergonomie et en psychologie du travail ». Le travail humain, 4(68), 289-316.

Dejours, C. (2009). Travail vivant, Tome 2 : Travail et émancipation. Paris : Payot.

Deville, J., Starck, S. (2013). Stages longs en entreprise : un dispositif original qui interroge la professionnalité enseignante dans le monde du travail. Les dossiers des sciences de l'éducation. 30, 85-100.

Kherroubi, M., \& Rochex, J.Y. (2004) La recherche en éducation et les ZEP en France.2. Apprentissages et exercice professionnel en ZEP : résultats, analyses, interprétations. Revue Française de Pédagogie, 146, 115-190

Leontiev, A. (1959). Le développement du psychisme. Éditions de l'Université de Moscou.

Leplat, J. (1997). Regards sur l'activité de travail. Contribution à la psychologie ergonomique. Paris, PUF.

Léger-Jarniou, C. (2008). Développer la culture entrepreneuriale chez les jeunes. Théories et pratiques. Revue Française de Gestion, 185(5), 161-174. 
Lorino, P. (2014). Développement des compétences et reconstruction de l'activité collective. In E. Bourgeois, S. Enlart (Dir.), Apprendre dans l'entreprise (pp. 207-220). Paris : PUF.

Loriol, M. (2000). Le temps de la fatigue. La gestion sociale du mal être au travail. Paris : Anthropos (Sociologiques).

Marcel, J.-F. (2009). De la prise en compte des pratiques enseignantes de travail partagé. Les Nouveaux Cahiers de la Recherche en Education, 12(1), 47-64.

Marcel, J.-F., Dupriez, V., \& Périssed-Bagnoud, D. (2007). Coordonner, collaborer, Oddone, I. (1981). Redécouvrir l'expérience ouvrière. Vers une autre psychologie du travail. Paris : Éditions sociales.

Marcel, J.-F., \& Garcia, A. (2010). Pratiques enseignantes de travail partagé et apprentissages professionnels. In L. Corriveau et al. (Eds.), Travailler ensemble dans les établissements scolaires et de formation (pp. 13-29). Bruxelles : De Boeck Supérieur « Perspectives en éducation et formation ».

Marcel, J.-F., \& Piot, T. (Dir.). (2005). Dans la classe, hors la classe, Lyon : INRP.

Pépin, M. (2011). L'entrepreneuriat en milieu scolaire, de quoi s'agit-il ? McGill Journal of Education / Revue des sciences de l'éducation de McGill, 46(2), 303-326. URI : http://id.erudit.org/ iderudit/1006441ar DOI : 10.7202/1006441ar

Piot, T. (2009). Quels indicateurs pour mesurer le développement professionnel dans les métiers adressés à autrui ? Questions Vives, 5(11).

coopérer. De nouvelles pratiques enseignantes. Bruxelles : De Boeck.

Schwartz, Y. (1987). Travail et usage de soi. In Je, sur l'individualité, Paris : Editions sociales.

Schwartz, Y. (Dir.). (1997). Reconnaissances au travail. Pour une approche ergologique, Paris : PUF, Le travail humain.

Six, F. (2002). De la prescription à la préparation du travail ; la dimension sociale du travail. Exemple du travail des compagnons et de l'encadrement sur les chantiers du bâtiment. In J.M. Evesque, A.M. Gautier, C. Revest, Y. Schwartz, J.L. Vayssière (Eds.), Les évolutions de la prescription (pp. 127-133), Actes du XXXVIII ${ }^{\mathrm{e}}$ congrès d'Aix-en-Provence : GREACT et SELF.

Starck, S., Champy-Remoussenard, P., Dumet T., Labbé, S., \& Zaid A. (2012). Les stages d'une année en entreprise d'enseignants du second degré, Recherche sur les relations écoles entreprises. Rapport de recherche, Lille, Université Lille 3.

Trognon, A., Dessagne, L., Hoch, R., Dammerey, C., \& Meyer, C. (2004). Groupes, collectifs et communications au travail. In E. Brangier, A. Lancry \& C. Louche (Eds.), Les dimensions humaines du travail : Théories et pratiques de la psychologie du travail et des organisations (pp. 415-449). Nancy : Presses Universitaires de Nancy.

Vergnaud, G. (1995). Évolution du travail et formation des compétences. Le Monde, mercredi 20 décembre 1995

Wittorski, R. (2007). Professionnalisation et développement personnel. Paris : L'harmattan, col. Savoirs et Actions.

Zaid, A. \& Champy-Remoussenard, P. (accepté). Extended business work placements for teachers : between lived experience and barriers to professionalization. European Journal of Teacher Education.

Zeitler, A. (2012) Apprentissages interprétatifs et construction de l'expérience. Recherche \& Formation, 70, 31-46. 


\section{NOTES}

1. Ces réflexions sont à poursuivre de manière plus avancée. Elles se basent sur une revue non systématique de la littérature et s'appuient uniquement sur des travaux en langue française.

2. C'est nous qui soulignons.

3. Les textes sont produits dans le cadre d'ateliers d'écriture et d'échanges sur l'activité, sur la base d'une méthode spécifique visant la production de récits en première personne relatant la singularité du vécu des situations de travail réel.

4. Imaginé et mis en œuvre par le Professeur G. Fath à l'occasion de ses enseignements en Licence de Sciences de l'Éducation à l'Université Nancy2.

5. Ces dispositifs s'inscrivent le plus souvent dans les éducations à, et dans un ensemble de dispositifs et démarche visant des objectifs spécifiques, en lien avec des «publics à besoin spécifiques » et/ou des problèmes sociaux. Ils engagent, pour la plupart d'entre eux des formes pédagogiques spécifiques et un partenariat entre différents professionnels chargés d'une mission éducative.

6. Dans le cadre d'un financement FSE (Fonds Social Européen) du projet IDEE (Innovons et Développons l'Esprit d'Entreprendre).

7. Les stages en entreprise par exemple (Champy-Remoussenard \& Starck (2013); Deville, Starck (2013) ; Starck, Deville (2014),

8. La terminologie relative à ces pratiques en plein développement n'est pas encore stabilisée par plus en France que dans d'autres pays (Léger-Jarniou, 2008, Pépin, 2011).

9. Ces résultats corroborent ceux recueillis lors de l'enquête sur les stages d'un an en entreprise d'enseignants d'économie-gestion. Ceux-ci relatent comment leur séjour dans les entreprises permet aux salariés de celles-ci de découvrir des compétences qu'ils n'auraient pas attribués aux enseignants avant de travailler avec eux (Champy-Remoussenard \& Deville, 2013).

10. Association qui est le partenaire historique et le plus actif.

\section{RÉSUMÉS}

Les recherches en analyse du travail montrent que les dimensions collectives constituent une caractéristique quasi universelle du travail humain que l'on peut donc considérer comme générique et transversale. Elles trouvent des déclinaisons spécifiques dans des secteurs d'activité et des «métiers » différents. A l'heure où le travail humain se transforme de façon considérable et accélérée, les dimensions collectives évoluent elles aussi et participent d'une transformation des métiers de la relation humaine, notamment des activités de ceux qui éduquent, forment, enseignent, accompagnent. En mobilisant les résultats des études qui portent sur les dimensions collectives dans l'ensemble des activités de travail, on peut renouveler la compréhension des activités des enseignants, notamment, dans leurs dimensions collectives en ce qu'elles recèlent de travail caché et difficilement formalisable. Nous montrerons tout d'abord que les dimensions collectives sont des dimensions génériques du travail humain, qu'elles participent de son caractère mal visible. Nous insisterons sur le statut qu'elles donnent à la relation à autrui, au lien qu'elles entretiennent avec le travail réel, aux processus de construction, de transmission, 
d'évaluation des compétences. C'est enfin sur cette base que la spécificité des dimensions collectives du travail des enseignants sera discutée, plus particulièrement dans les dispositifs « partenariaux » qui reconfigurent les formes traditionnelles du travail des enseignants.

The research work analysis shows that collective dimensions are an almost universal feature of human labor that can therefore be considered as a generic and cross. They are variations in specific industries and different "professions". At a time when human labor changes significantly and fast, collective dimensions are also changing and part of a transformation of human relations professions, including those activities that educate, train, teach, accompany. Mobilizing the results of studies on collective dimensions in all work activities, can be renewed understanding of the activities of teachers, especially in their collective dimensions in that they contain hidden work and difficult to formalize. We first show that collective dimensions are generic dimensions of human labor, they are part of the visible evil character and its educational function. We will insist on the status they give to relationships with others, the relationship they have with the actual work, the construction process, transmission, assessment of skills. Finally, it is on this basis that the specificity of the collective dimensions of teachers' work will be discussed, particularly in the "partnership" devices that challenge the more traditional forms of teachers' work.

\section{INDEX}

Keywords : partnership teaching activities, work analysis, collective dimensions, human labor Mots-clés : activités d'enseignement partenariales, analyse du travail, dimensions collectives du travail, travail humain

\section{AUTEUR}

\section{PATRICIA CHAMPY-REMOUSSENARD}

Professeur, Université Lille 3, Proféor-CIREL (EA 4354) 